\title{
Toward Continuous Deracemization via Racemic Crystal Transformation Monitored by in Situ Raman Spectroscopy
}

\author{
Christos Xiouras, ${ }^{*} \dagger \odot$ Giuseppe Belletti, ${ }^{\ddagger}$ Raghunath Venkatramanan, ${ }^{\S}$ Alison Nordon,

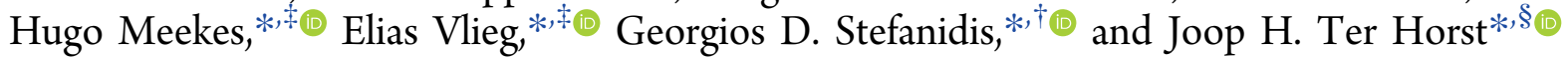

${ }^{\dagger}$ Process Engineering for Sustainable Systems (ProcESS), Department of Chemical Engineering KU Leuven, Celestijnenlaan 200F, 3001 Leuven, Belgium

${ }^{\ddagger}$ Institute for Molecules and Materials, Radboud University, Heyendaalseweg 135, 6525 AJ Nijmegen, The Netherlands

${ }^{\S}$ EPSRC Centre for Continuous Manufacturing and Crystallisation (CMAC), Strathclyde Institute of Pharmacy and Biomedical Sciences (SIPBS), Technology and Innovation Centre, University of Strathclyde, 99 George Street, Glasgow G1 1RD, U.K.

"WestCHEM, Department of Pure and Applied Chemistry and Centre for Process Analytics and Control Technology, University of Strathclyde, Glasgow G1 1XL, U.K.

Supporting Information

ABSTRACT: In this work, we demonstrate a semibatch solid-state deracemization process for $N$-(2chlorobenzylidene)henylglycine amide (NCPA), a complex chiral polymorphic system that involves three types of crystalline racemates (racemic compound and conglomerate forms I and II). In this process, gradually fed metastable racemic compound crystals are converted in situ to crystals of the preferred (seeded) enantiomer under grinding conditions through a series of solvent-mediated transformations in a

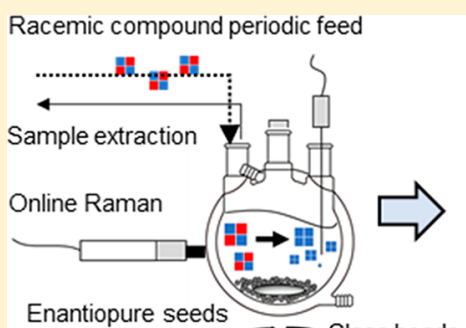

Enantiopure seeds $\subset \geq$ Glass beads

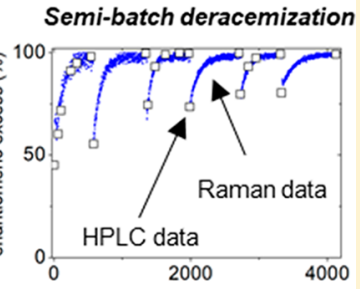

Time (min) racemizing solution. The phase diagram for this system shows that while conglomerate form II is stable under the conditions examined (acetonitrile at $21^{\circ} \mathrm{C}$ ), form I crystals of a single enantiomer (used as seeds) are unstable at (nearly) racemic compositions and convert to the racemic compound upon addition of the racemization catalyst. Thus, care needs to be exercised in order to fully convert form I to form II before addition of the racemization catalyst in order to prevent the undesired crystallization of the racemic compound. This can be achieved by adding a small amount of water, which is found to enhance the nucleation and growth kinetics of the most stable conglomerate form II, eventually leading to complete deracemization. Importantly, we show that this special deracemization process can be easily monitored online by Raman spectroscopy, which gives access to the evolution of the solid-phase composition. For the studied system, this information can in turn be used to directly estimate the solid-phase enantiomeric excess online throughout the process, as long as conglomerate crystals of the counter enantiomer do not form.

\section{INTRODUCTION}

In the past decade, solid-state deracemization processes such as Viedma ripening $^{1}$ and temperature cycling-enhanced deracemization $^{2}$ have gained considerable attention as feasible means to obtain enantiopure solid products. ${ }^{3-11}$ Most of these processes are typically carried out in laboratory-scale batch mixed crystallizer vessels, where a population of conglomerate crystals close to the racemic composition is suspended in a (nearly) saturated solution in which fast solution-phase racemization occurs. Under these conditions, intense grinding ensured by the presence of grinding media or programmed heating and cooling cycles in the suspension drive the solid phase to a homochiral end state (enantiomeric purity $>99.9 \%$ ) in an autocatalytic fashion. While the exact mechanisms under which this occurs have been debated, ${ }^{3-6}$ the method is generally very reliable, requires little information prior to its application, and is in theory very productive, as the amount of enantiopure solid obtained in a single batch is not limited by the solubility of the compound in the solvent. The feasibility of deracemization processes has now been demonstrated for a plethora of chiral compounds, such as amino acids ${ }^{7,8}$ and their derivatives, ${ }^{9-11}$ pharmaceutical intermediates, ${ }^{12,13}$ and organometallic complexes, ${ }^{14}$ while their application in compounds with more than one chiral center was also recently realized. ${ }^{15}$

Crucial prerequisites for any successful deracemization process are facile solution-phase racemization and conglomerate crystallization (each enantiomer forming individual crystals). ${ }^{12}$ However, the majority of chiral molecules, when they are crystallized from racemic mixtures, form racemic compounds (i.e., individual crystals that contain both enantiomers in equal amounts). The racemic compound and conglomerate crystals possess different crystal structures and in the presence of fast

Received: July 3, 2019

Revised: August 22, 2019

Published: August 23, 2019 
solution racemization can be considered as polymorphs, ${ }^{16}$ allowing the possibility for interconversion between the two forms through solvent-mediated crystal transformations. ${ }^{17}$ In our previous works, ${ }^{18,19}$ we have shown independently that combining such a transformation of a racemic compound to a conglomerate with Viedma ripening, despite the presence of the racemic compound, can still lead to enantiopurity. Using this transformation, remarkably, the rate of deracemization was substantially increased (by a factor of 10) in comparison to "conventional" Viedma ripening.

Simulations of this special deracemization process ${ }^{20}$ revealed that enantiopurity is attained through a combination of preferential crystallization in the presence of a racemization agent (also termed second-order asymmetric transformation, SOAT $^{17}$ ) and Viedma ripening. The dominant deracemization mechanism depends heavily on the crystal transformation kinetics ${ }^{21}$ and is determined by the extent of nucleation of the counter enantiomer. If the latter does not occur, deracemization proceeds purely by SOAT, leading to the fastest deracemization possible, while in the case of counter enantiomer formation, Viedma ripening is required to reach enantiomeric purity, leading to a slower process. ${ }^{20}$ In the same study, it was shown theoretically that, if the racemic compound is highly metastable with respect to the conglomerate, the amount of racemic compound added must be carefully managed in order to control the supersaturation generated and prevent nucleation of the counter enantiomer. Therefore, a continuous or semicontinuous operating mode, where the racemic compound is gradually added to the suspension containing seeds of the preferred enantiomer, may be advantageous to enhance the process productivity and would constitute an important step toward the development of continuous deracemization processes. The benefits of in situ gradual feeding in Viedma ripening were also demonstrated in a process where the compound to be deracemized was generated in situ by a chemical reaction. ${ }^{13}$ Such a process was shown to achieve a higher deracemization rate in comparison to conventional Viedma ripening (which starts at a nearly racemic composition), since it avoids operation at low enantiomeric excess regions where the deracemization rate is slow.

Process analytical technologies (PATs) have become integral in the operation of (semi)continuous crystallization processes, as they enable real-time monitoring of critical process parameters and can be used to develop feedback control strategies and automation. ${ }^{22}$ However, for chiral resolution and deracemization processes this has not yet been possible, as the PATs currently available are chirally blind and cannot differentiate between the enantiomer solutes and crystals. The traditional analytical methods that yield the enantiomeric excess, and thus the evolution of the process, rely on manual sampling of the solid phase and sample preparation followed by chiral HPLC and/or polarimetry. On the other hand, online Raman spectroscopy, ${ }^{23}$ although being chirally blind as well, may be suitable for the in situ monitoring of the crystal transformation/ deracemization process, since Raman can detect the specific lattice vibrations for different crystal structures. ${ }^{24}$ Hence, Raman is capable of distinguishing between enantiomers packed in different crystal structures (racemic compounds, conglomerates). ${ }^{25}$ Although several authors have reported the ability of monitoring the solid-phase fractions of different polymorphs during solvent-mediated transformations using online Raman spectroscopy, ${ }^{26-31}$ to our knowledge there has been no previous work reported using Raman to quantitatively determine the compositions of racemic and conglomerate crystals of chiral compounds undergoing a dissolution/crystallization process. In addition, chemometric treatments can be applied to the Raman spectra, ${ }^{32-34}$ allowing accurate monitoring of the solid-phase composition when more than two solid phases are involved. $^{35-37}$

In this work, we present a semibatch deracemization process for chiral $\mathrm{N}$-(2-chlorobenzylidene) phenylglycine amide (NCPA), which is based on the conversion of racemic crystals fed to a racemizing suspension containing seed crystals of the preferred enantiomer in the presence of grinding. Furthermore, through estimation of the phase diagram, we determine regions of stability for the three solid phases involved (two conglomerates and a racemic compound) and we reveal the crucial effect of water content on the outcome of the crystal transformations between the solid phases, which ultimately affects the deracemization process. Finally, we demonstrate for the first time the relevance and applicability of Raman spectroscopy for the online monitoring of this special deracemization process.

\section{EXPERIMENTAL SECTION}

2.1. Synthesis of Starting Materials. $N$-(2-Chlorobenzylidene)phenylglycine amide (NCPA) was used as a model compound in this study, which according to George et al. ${ }^{38}$ crystallizes in three forms, two of which are conglomerates and one is a racemic compound. Racemic NCPA and enantiopure R-NCPA were synthesized after slight modifications of a reported procedure. ${ }^{39}$ The details of the chemical synthesis and isolation of NCPA as a racemic compound and conglomerates I and II are reported in the Supporting Information.

2.2. Solubility Measurements. The solubility of the three solid phases of NCPA was determined in various acetonitrile/water mixtures in the presence and absence of the racemization catalyst DBU at the experimental temperature of $21^{\circ} \mathrm{C}$. A known amount $(\sim 0.1 \mathrm{~g})$ of pure NCPA of the desired phase (conglomerate I or II or the racemic compound) was weighed in a $5 \mathrm{~mL}$ glass vial equipped with a magnetic stirrer, and a known amount ( $\sim 1.6 \mathrm{~g})$ of solvent was added. The vials were placed inside a Crystalline apparatus (Technobis B.V.) and were stirred at $1200 \mathrm{rpm}$ for $48 \mathrm{~h}$ at $21^{\circ} \mathrm{C}$ to ensure attainment of a solidliquid equilibrium, after which the solubility was determined gravimetrically. Each measurement was conducted at least twice, and the relative deviation was less than $5 \%$. The resulting solid phase was subsequently analyzed via Raman spectroscopy to confirm that crystal transformation had not taken place.

2.3. Deracemization Experiments. Figure 1 (top) presents the experimental setup used for all deracemization experiments by conversion of the racemic compound to a conglomerate, which consists of a jacketed $30 \mathrm{~mL}$ round-bottom flask containing a total solution volume of about $20 \mathrm{~mL}$. All experiments were conducted at $21^{\circ} \mathrm{C}$, and samples were stirred at $500 \mathrm{rpm}$ using a magnetic stirring bar (length 2.5 $\mathrm{cm}, \varnothing 1 \mathrm{~cm})$ in the presence of $10 \mathrm{~g}$ of glass beads $(\varnothing 2 \mathrm{~mm})$ to enable particle breakage. The reactor was brought in external contact with a Raman probe, as seen in Figure 1, to allow in situ measurements of the Raman spectra in the suspension through the glass and the reactor jacket. This configuration proved advantageous in comparison to positioning direct contact probes in the suspension that can be damaged by the glass beads and are impractical on a small scale, but the spectra recorded required processing to reduce the background interference caused by the presence of the glass. Figure 1 (bottom) shows the racemization reaction of NCPA enabled by catalytic amounts of diazabicyclo[5.4.0] undec-7-ene (DBU).

2.3.1. Batch Experiments. For the batch deracemization experiments, a known amount of R-NCPA (form I) was mixed with a known amount of RS-NCPA (racemic compound) and placed in the reactor together with the glass beads $(10 \mathrm{~g})$. Subsequently, $20 \mathrm{~mL}$ of acetonitrile was placed in the reactor, stirring was initiated, and the Raman measurements were started (with a delay of about $8 \mathrm{~min}$ ) to 


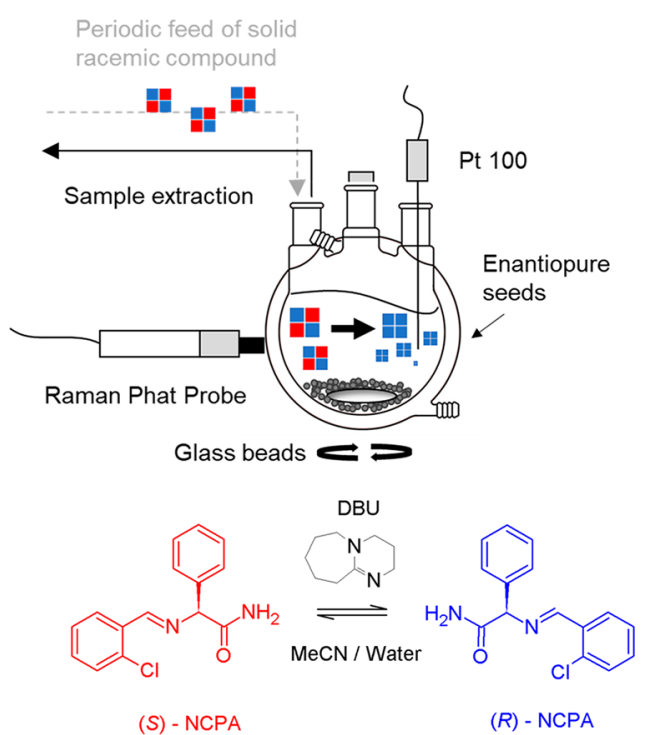

Figure 1. (top) Schematic description of the experimental setup used in this study and the basic concept for deracemization: gradually fed metastable racemic compound crystals (red-blue blocks) are converted in situ to crystals of a single chirality (blue blocks) through solvent-mediated crystal transformation under racemizing conditions. (bottom) Racemization reaction of $\mathrm{N}$-(2-chlorobenzylidene)phenylglycine amide (NCPA) in the presence of catalytic 1,8diazabicyclo[5.4.0]undec-7-ene (DBU).

monitor the time evolution of the solid-phase composition. After 17 min, $0.2 \mathrm{~mL}$ of DBU was added to initiate racemization. For some experiments, small amounts of water $(0.65-3.9 \mathrm{~mL})$ were added immediately after the addition of the acetonitrile.

2.3.2. Semibatch Experiments. For the semibatch deracemization experiments, initially the same procedure was followed as for the batch experiments. After startup, the racemic compound was fed periodically to the reactor by opening the reactor and manually adding a known amount of dry RS-NCPA (racemic compound). Together with the racemic compound, small amounts of water were added as well.

2.3.3. Sampling. In all experiments, suspension samples were periodically extracted using a pipet $(0.2 \mathrm{~mL})$ and the solid was filtered over preweighed paper filters, washed using $1 \mathrm{~mL}$ of diisopropyl ether, and oven-dried overnight at $30^{\circ} \mathrm{C}$ under vacuum. After a determination of the solid mass, the solids were subjected to offline Raman spectroscopy to determine the weight fractions of the various phases ( $X_{\mathrm{RAC}}, X_{\mathrm{I}}$, and $X_{\mathrm{II}}$ for the racemic compound and forms I and II, respectively) and enantiomeric excess, $E$, determination via chiral HPLC. After the end of each experiment, the solids in the reactor were collected and dried to determine the solid yield, $y$, according to

$$
y=\frac{m_{\mathrm{r}}}{m_{\mathrm{t}}-C_{\mathrm{II}}^{*} m_{\mathrm{s}}}
$$

where $m_{\mathrm{r}}$ is the mass recovered at the end of the experiment, $m_{\mathrm{t}}$ is the total mass of the solids added, $m_{\mathrm{s}}$ is the mass of the solvent mixture and $C_{\mathrm{II}}^{*}$ is the solubility of conglomerate form II at $21^{\circ} \mathrm{C}$ in the respective solvent mixture.

2.4. Online Quantification of Solid-Phase Fractions by Raman Spectroscopy. Raman spectra were collected through the reactor glass jacket via contactless in situ measurements (Figure 1 using a process Raman analyzer (Kaiser RXN2, PhatProbe) spectrometer at $785 \mathrm{~nm}$ laser excitation. The spectra were captured with an exposure time of $10 \mathrm{~s}$ and 6 accumulations over $2-3 \mathrm{~min}$. A laser spot diameter of $6 \mathrm{~mm}$ was used so that sufficient solid mass was sampled in each acquisition to reliably detect the different solid forms suspended in the liquid. Offline spectra were taken as well for filtered solid samples from the suspension using the same method in order to compare with the in situ data. All raw spectra files were pretreated using MATLAB by first taking the second derivative of each spectrum with respect to wavelength via the Savitzky-Golay algorithm using a second-order polynomial, a seven-point smoothing window, and standard normal variate (SNV) transformation, to reduce baseline shift effects and to increase peak resolution and deconvolution. ${ }^{40}$

For quantification of the solid-phase fractions, a calibration model was developed (see the Supporting Information for the details). For this purpose, 30 ternary solid mixtures of the three forms of NCPA having various known solid compositions were prepared and partial leastsquares (PLS) regression analysis was performed in MATLAB (SIMPLS algorithm ${ }^{41}$ ) on the pretreated calibration spectra using three latent variables (LVs). It was found that the first three LVs are sufficient to explain $99.53 \%$ of the total variance in the data and afford a minimum root-mean-square error of prediction (RMSEP). In order to assess the predictive capabilities of the calibration model, an independent validation set of four samples was prepared. Table S1 in the Supporting Information shows the actual versus predicted values of the mass fractions of the three solid phases giving RMSEP values of $1.93 \%, 1.58 \%$, and $0.79 \%$ for the racemic compound and forms I and II, respectively, which were considered sufficient in the present study.

Strictly speaking, this calibration approach is only valid for offline analysis of dried solid samples. It is known that, for accurate in situ monitoring using Raman, it is best to prepare the calibration samples under conditions as close to those of the experiments as possible ${ }^{23,33}$ (in a stirred suspension at the right process temperature and suspension density, with additional parameters such as particle size and shape), as the Raman spectra can be infuenced by varying conditions, and peaks due to the solvent would appear in the spectra. However, taking accurate calibration spectra in suspensions is challenging when fast solvent-mediated transformations occur and models accounting for the effects of suspension density and particle size/shape together with solid-phase composition for more than two phases can be quite complex. For this reason, we decided to assess the capability of extending the present (offline) calibration to online measurements. This was done by applying the same data preprocessing and prediction procedure for offline and online measurements, which showed good overall agreement (see the Supporting Information for a comparison of the offline and online data for all reported experiments). It is noted that, in order for this to hold, care must be taken to exclude spectral regions from the analysis that are influenced by the presence of additional compounds such as the solvent, glass beads and wall, and racemization catalyst.

2.5. HPLC Analysis for Determination of the Solid-Phase Enantiomeric Excess. All dried solid samples were dissolved in ethanol, and their enantiomeric excesses were determined by chiral HPLC analysis using an Agilent 1290 UPLC apparatus. The enantiomers of NCPA were separated in a Phenomenex Lux $3 \mu \mathrm{m}$ iCellulose- 5 column running at $0.5 \mathrm{~mL} / \mathrm{min}$ of ethanol at ambient temperature and were detected by a diode array detector (DAD) at 254 $\mathrm{nm}$. The injection volume was $4 \mu \mathrm{L}$, and the retention times were 2.4 and $2.9 \mathrm{~min}$ for the $S$ and $R$ enantiomers, respectively. The enantiomeric excess was calculated on the basis of the integrated peak areas according to $E=\left(A_{R}-A_{S}\right) /\left(A_{R}+A_{S}\right)$.

\section{RESULTS}

3.1. Solubility and Phase Diagrams. In order to design a deracemization process employing $R$ (form $\mathrm{I}$ ) seeds and racemic compound crystals, we first measured the solubility of the three crystalline phases of NCPA in acetonitrile containing $1.27 \mathrm{wt} \%$ DBU (Table 1). Under these fast solution racemization conditions, conglomerate crystals and racemic crystals behave similarly to polymorphs, and transformations between the phases are possible. ${ }^{19}$ It is seen that at $21{ }^{\circ} \mathrm{C}$ in acetonitrile, the order of solubility is form I > racemic compound > form II. Conglomerate form II, being $13 \%$ less soluble than the racemic compound, is therefore the most stable form at $21{ }^{\circ} \mathrm{C}$.

On the basis of the solubilities of the phases reported in Table 1 and assuming the validity of Meyerhoffer's double-solubility rule ${ }^{17}$ for the conglomerates and a solubility product of $\left(C_{R} C_{S}\right)^{*}$ 
Table 1. Solubilities of the Solid Phases of NCPA under Fast Racemization Conditions (1.27 wt \% of DBU in Acetonitrile at $21^{\circ} \mathrm{C}$ )

$\begin{array}{llc}\text { NCPA solid phase } & & \text { solubility (mg/g solvent) } \\ \text { form I } & C_{1}^{*} & 42.5 \\ \text { form II } & C_{\mathrm{II}}^{*} & 35.6 \\ \text { racemic compound } & C_{\mathrm{RAC}}^{*} & 40.8\end{array}$

$=\left(C_{\mathrm{RAC}}^{*} / 2\right)^{2}$ for the racemic compound, ${ }^{17}$ the phase diagram at $21{ }^{\circ} \mathrm{C}$ in acetonitrile is deduced and reported in Figure 2 . It is
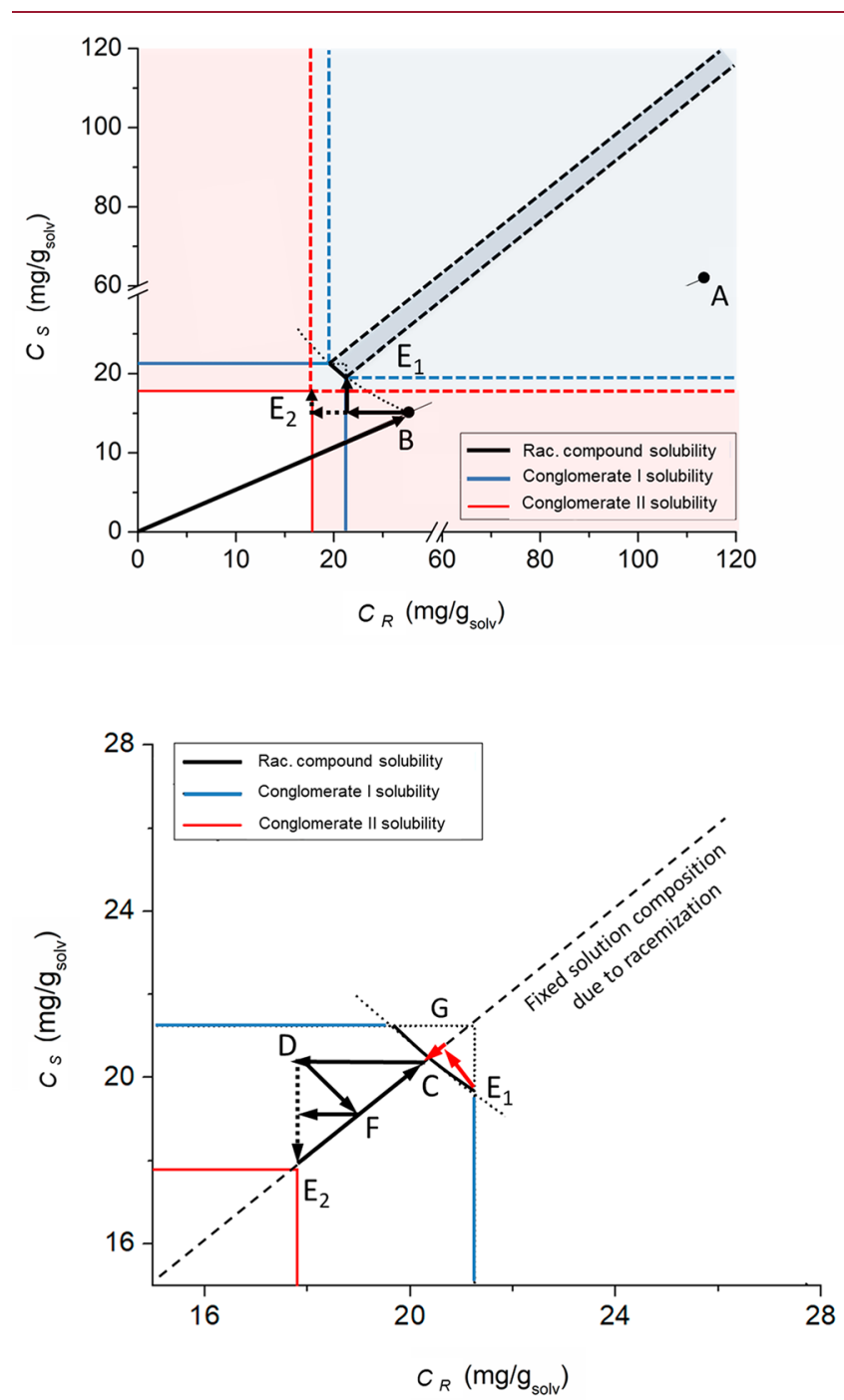

Figure 2. Phase diagram of $R$ - and $S$-NCPA at $21{ }^{\circ} \mathrm{C}$ in acetonitrile without (top) and with racemization (bottom). Lines $\mathrm{E}_{2} \mathrm{CDF}$ and $\mathrm{E}_{1} \mathrm{GC}$ in the bottom figure represent the deracemization process by conversion of the racemic compound to conglomerate II and the undesirable conversion of conglomerate I to the racemic compound, respectively.

noted that since the racemization catalyst (DBU) was not found to significantly affect the solubilities, this phase diagram can be used to describe and interpret transformation behavior both with and without the racemization catalyst. In the absence of the racemization catalyst and given enough time, a suspension of conglomerate form II will equilibrate with the solution, since form II is the most stable form. Depending on the overall composition, this suspension contains a mixture of both enantiopure crystals or only crystals of a single handedness (light blue shaded region and red shaded regions in Figure 2 top, respectively). On the other hand, the racemic compound is only slightly more stable than conglomerate form I. Thus, in the absence of form II and provided that the crystal transformation kinetics to form II are slow, there exists a rather narrow compositional region in which a suspension of only racemic compound crystals is in a metastable equilibrium with the solution (dark blue shaded region in Figure 2 top).

In order to study the possibility for deracemization in this complex system, a series of experiments were designed in which a mixture of form I crystals of the $R$ enantiomer and crystals of the racemic compound were slurried together in acetonitrile (21 ${ }^{\circ} \mathrm{C}$ ) under grinding conditions in the presence of small amounts of water. An overview of the conditions of the experiments performed is presented in Table 2.

Experiments B2-B4 are all started using the same solid content comprised of $70.6 \%$ racemic compound and $29.4 \%$ of the $R$ enantiomer (form I) corresponding to point A in Figure 2 (top). Since the racemization catalyst is not present initially, two scenarios are envisaged for these experiments on the basis of the phase diagram in Figure 2. In the first scenario, the stable form II does not form; thus, dissolution occurs until the solution composition reaches the metastable eutectic point $\mathrm{E}_{1}$ (liquid phase enantiomeric excess $E=4.1 \%)$, leading to enrichment of the solids in the $R$ enantiomer (63\% racemic compound and $37 \%$ conglomerate I). In the second scenario, the stable form II does form and the solution composition shifts toward the stable eutectic point $\mathrm{E}_{2}(E=0 \%)$.

Figure 2 (bottom) shows what happens in the two scenarios once the racemization catalyst is added $17 \mathrm{~min}$ after the start of these experiments. In the first scenario, in the absence of stable form II, the solution composition at point $\mathrm{E}_{1}$ is enriched with $R$ and therefore, in the presence of the racemization catalyst, conversion of the excess $R$ enantiomer to the $S$ enantiomer will occur (line $E_{1} G$ ). At point $G$, the solution is supersaturated with respect to the racemic compound, which induces its crystallization and the concentration in the solution following line GC. Since at point $\mathrm{C}$ the solution is now undersaturated with respect to form $I R$ crystals, further dissolution of the $R$ form I crystals occurs, followed again by racemization and crystallization of the racemic compound until all $R$ form I crystals convert to the racemic compound.

Adding racemization catalyst during the second scenario when $R$ crystals of stable form II have formed and the solution composition is closer to point $\mathrm{E}_{2}$, dissolution of the racemic compound will occur (line $\mathrm{E}_{2} \mathrm{C}$ ), followed by crystallization of $R$ form II crystals (line $\mathrm{CD}$ ) and eventual conversion of $S$ molecules to $R$ through the racemization to remove the enrichment of $S$ (diagonal line DF). This in turn causes further crystallization of the $R$ enantiomer, which is again followed by racemization and so forth, overall bringing the solution composition gradually closer to $E_{2}$. Note that, depending on the racemization kinetics, the solution composition could alternatively follow directly dashed line $\mathrm{DE}_{2}$, which corresponds to the crystallization of the $S$ form II crystals. Near point $\mathrm{E}_{2}$ the solution is again undersaturated with respect to the racemic compound; thus, the process repeats until all of the racemic compound crystals convert to form II crystals.

Thus, in Figure 2, bottom, the line $\mathrm{E}_{2} \mathrm{CDF}$ represents the deracemization process, possible through the transformation of the racemic compound to stable form II and the fast 
Table 2. Overview of the Process Conditions in the Deracemization Experiments ${ }^{a}$

\begin{tabular}{|c|c|c|c|c|c|c|c|c|c|}
\hline expt & $X_{\mathrm{RAC}}($ wt $\%)$ & $X_{\mathrm{I}}($ wt $\%)$ & total solid $m_{\text {tot }}(\mathrm{g})$ & water $(\mathrm{g})$ & beads $(g)$ & $\mathrm{DBU}(\mathrm{g})$ & solubility of cong II $C_{\mathrm{II}}^{*}(\mathrm{mg} / \mathrm{g}$ solvent $)$ & yield $y(\%)$ & final $E$ of $R(\%)$ \\
\hline B1 & 80.0 & 20.0 & 4.00 & 0.00 & 10.0 & 0.2 & 35.6 & 75.0 & 3.0 \\
\hline $\mathrm{B} 2$ & 70.6 & 29.4 & 2.72 & 0.65 & 10.0 & 0.2 & 43.2 & 60.0 & 42.0 \\
\hline B3 & 70.6 & 29.4 & 2.72 & 1.30 & 10.0 & 0.2 & 48.2 & 81.0 & 100 \\
\hline B4 & 70.6 & 29.4 & 2.72 & 2.60 & 10.0 & 0.2 & 48.3 & 60.0 & 100 \\
\hline SB1 & $70.6^{b}$ & $29.4^{b}$ & $6.56^{b}$ & $3.90^{b}$ & 10.0 & 0.6 & 48.6 & 78.3 & 100 \\
\hline SB2 & $54.8^{b}$ & $45.2^{b}$ & $6.56^{b}$ & $3.90^{b}$ & 10.0 & 0.6 & 48.6 & 79.7 & 100 \\
\hline
\end{tabular}

${ }^{a}$ The metastable form I seeds were of the $R$ enantiomer. In all experiments, DBU was added $17 \mathrm{~min}$ after the start of the experiment and the mass of acetonitrile added was in all cases $15.72 \mathrm{~g}(20 \mathrm{~mL}) .{ }^{b}$ Total solid and water for the semibatch experiments is after all additions, while the mass fractions $X_{\mathrm{I}}$ and $X_{\mathrm{RAC}}$ are the initial ones.

B1

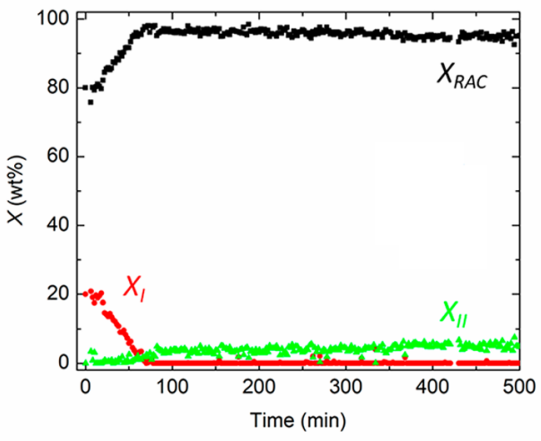

B3

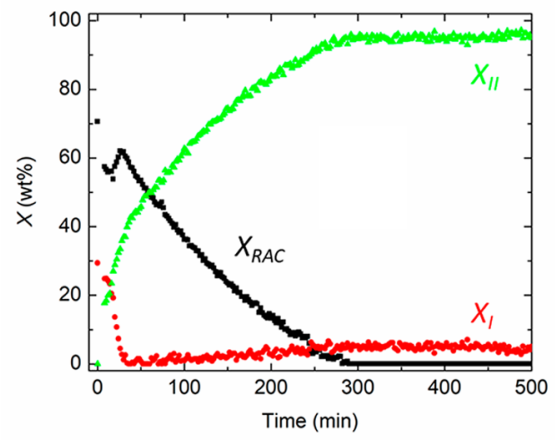

B2

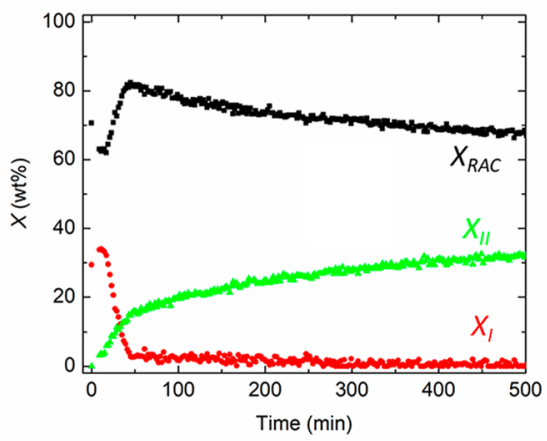

B4

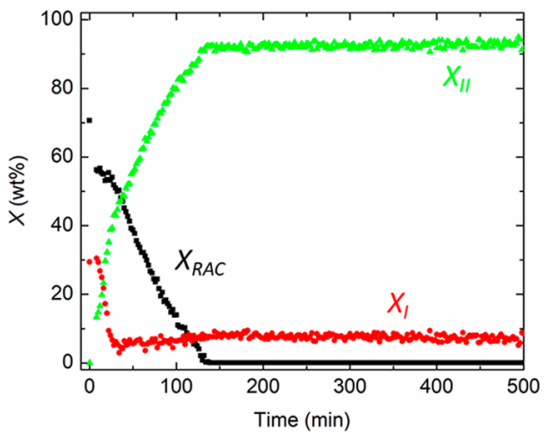

Figure 3. In situ Raman monitoring of the temporal evolution of the solid-phase fractions of NCPA in batch deracemization experiments (B1-B4, Table 2), starting with mixtures of $R$ form I crystals and racemic compound in acetonitrile/water mixtures at $21^{\circ} \mathrm{C}$ in the presence of DBU under grinding conditions. Red, green, and black points refer to form I $\left(X_{\mathrm{I}}\right)$, form II $\left(X_{\mathrm{II}}\right)$, and the racemic compound $\left(X_{\mathrm{RAC}}\right)$, respectively.

racemization, while line $\mathrm{E}_{1} \mathrm{GC}$ represents the undesirable transformation of metastable form I to the racemic compound. In order to prevent the latter transformation, care must be taken to have stable form II crystals present in the system. Therefore, in starting an experiment with single-enantiomer seeds of the metastable form I, the racemization catalyst should be added only later to allow some time for the formation of the stable form II crystals. It is noted that since stable form II seeds of the preferred enantiomer were initially unavailable, it was deemed necessary to start most experiments using single-enantiomer seeds of the metastable form I.

3.2. Effect of Water. The evolution of the solid-phase composition for experiments B1-B4, measured by Raman spectroscopy, are presented in Figure 3. Experiments B2-B4 were conducted under the same conditions, with the only difference being the amount of water added in the mixture. Despite its higher amount of solid and slightly different initial composition, experiment B1 without any added water is included in this series of experiments B1-B4. For all of these experiments the racemization catalyst was added only after 17 $\min$.

As seen in Figure 3, regardless of the amount of water, metastable form I disappears almost completely within $\sim 40 \mathrm{~min}$ for experiments B2-B4. In experiment B1 (Figure 4, top left), where no water is added, form I also disappears, but in a slower fashion than in cases B2-B4. Additionally, in B1, form II does not form at all within the first $17 \mathrm{~min}$ before the catalyst is added, while after catalyst addition it is only forming very slowly (Figure 2 , top left). Addition of the catalyst triggers the racemization of the excess R-NCPA molecules in the solution and the subsequent conversion to the racemic compound (line $\mathrm{E}_{1} \mathrm{GC}$ in Figure 3 bottom). Finally, in the absence of added water, the racemic compound is very slowly converting to form II, with the conversion being negligible even after $48 \mathrm{~h}$ (not shown here). HPLC indicates that no deracemization occurs under these conditions.

Conversely, in cases where water is added to the mixture (B2-B4), the formation of form II is accelerated substantially, 


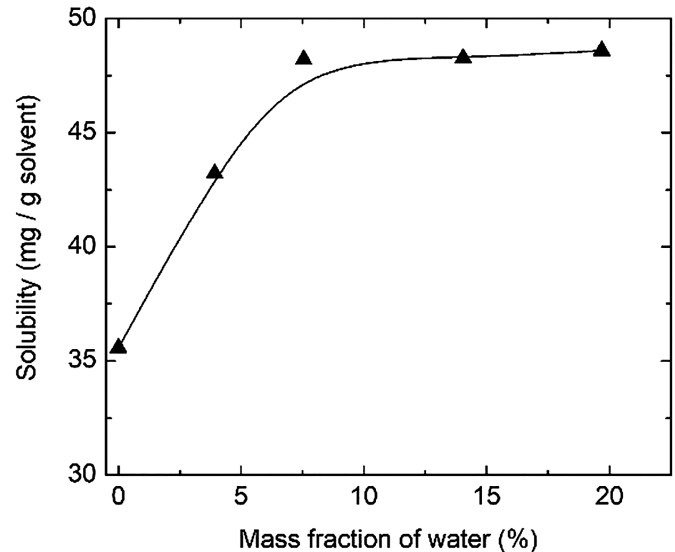

Figure 4. Solubility of NCPA conglomerate form II in various acetonitrile/water/DBU mixtures at $21^{\circ} \mathrm{C}$. The line is a guide to the eye.

with the acceleration being highly dependent on the amount of water present. Thus, when the racemization catalyst is added after $17 \mathrm{~min}$, the form II fractions are already 5.9\%, 27\%, and $29.6 \%$ for experiments B2-B4, respectively. In particular, for experiment B4, the metastable form I to stable form II transformation is almost complete when the catalyst is added; thus, no crystallization of the racemic compound is observed with its fraction decreasing monotonically. For experiments B2 and B3, addition of the catalyst leads to some conversion of the residual form $\mathrm{I}$ to the racemic compound (Figure 2, line $\mathrm{CE}_{3}$ ), but since a substantial amount of form II has already formed, the conversion to form II progresses further. Finally, for experiments B3 and B4, all of the solid phase transforms to the stable form II within 290 and $140 \mathrm{~min}$, respectively.

In order to obtain further insight into the effect of water, we measured the solubility of conglomerate form II in various acetonitrile/water mixtures at $21{ }^{\circ} \mathrm{C}$ corresponding to the conditions of experiments B1-B4. While the solubilities of the racemic compound and conglomerate form I could not be measured reliably in the mixed solvent system due to the fast transformations, it is expected thermodynamically that the relative solubility differences will be similar to those in the pure acetonitrile system, reported in Table $1 .{ }^{42,43}$ Thus, while the presence of water would affect the actual values of the phase diagram reported in Figure 2, the qualitative behavior is expected to be similar. The solubility results of NCPA conglomerate form II as a function of water content are shown in Figure 4.

Although NCPA is hardly soluble in pure water, the NCPA solubility in acetonitrile increases upon increasing the water mass fraction to $\sim 7.5 \%$, after which the solubility reaches a plateau value of $\sim 48 \mathrm{mg} / \mathrm{g}$ solvent, which pertains up to water fractions of $20 \%$. Such appearances of maxima in solubility are not uncommon for organic compounds in mixed organic/ aqueous solvents but have a complex thermodynamic basis arising from both enthalpy and entropy effects and have not yet been fully described. ${ }^{44}$ Nevertheless, the increase in solubility may partially explain the enhancement in the rate of the crystal transformations upon water addition, since a higher solubility leads not only to enhanced mass transfer but also to lower interfacial tension and thus a lower free energy barrier for the nucleation of the stable form II. ${ }^{45}$ Although further research is needed to elucidate the exact effect of water, the results suggest that the nucleation and growth kinetics of the stable form II are substantially increased by the amount of water added. In addition to an increase in the solubility, a protic solvent such as water could also lead to degradation of NCPA (e.g., by hydrolysis of the imine bond). In order to confirm the chemical stability of NCPA in the presence of water, an additional experiment was performed (see the Supporting Information), in which pure NCPA was slurried for $48 \mathrm{~h}$ in an acetonitrilewater-DBU system at room temperature. ${ }^{1} \mathrm{H}$ NMR spectra confirmed that no such degradation of NCPA occurs under the experimental conditions relevant to this work.

3.3. Online Estimation of the Enantiomeric Excess from Raman Data. The in situ Raman data collected for experiments $\mathrm{B} 1-\mathrm{B} 4$ can be used to monitor the evolution of the solid-phase enantiomeric excess, provided that the forms present (either I or II) remain a single enantiomer: i.e., no crystallization of the counter enantiomer crystals occurs. Then, the Ramanbased enantiomeric excess can be simply calculated as the sum of the form I and II fractions present at any given time. Figure 5 (left) presents a comparison of the sum of the form I and II
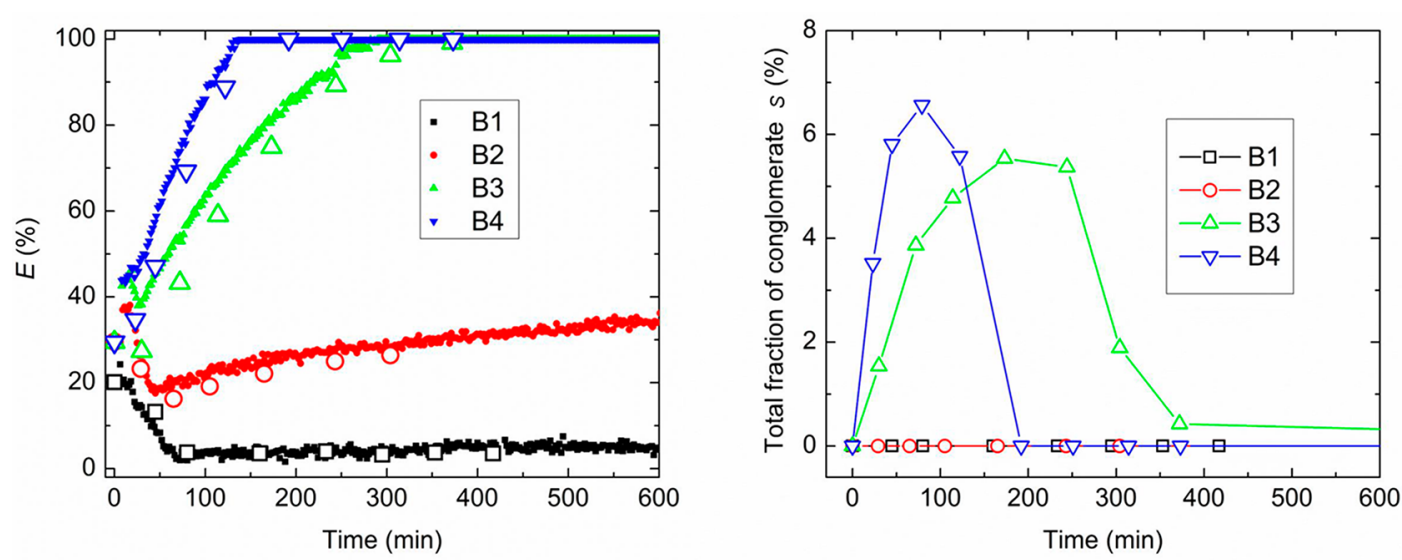

Figure 5. (left) Evolution of solid-phase enantiomeric excess during deracemization experiments, starting with mixtures of $R$ form I crystals and the racemic compound in acetonitrile/water mixtures at $21^{\circ} \mathrm{C}$ in the presence of DBU under grinding conditions. Filled symbols are enantiomeric excess estimates from the Raman measurements assuming the absence of counter enantiomer nucleation, while open symbols are the enantiomeric excess values measured offline by chiral HPLC. See Table 2 for the experimental conditions of experiments B1-B4. (right) Fraction of the total solid present as counter enantiomer $(S)$ crystals for the same experiments. 
SB1
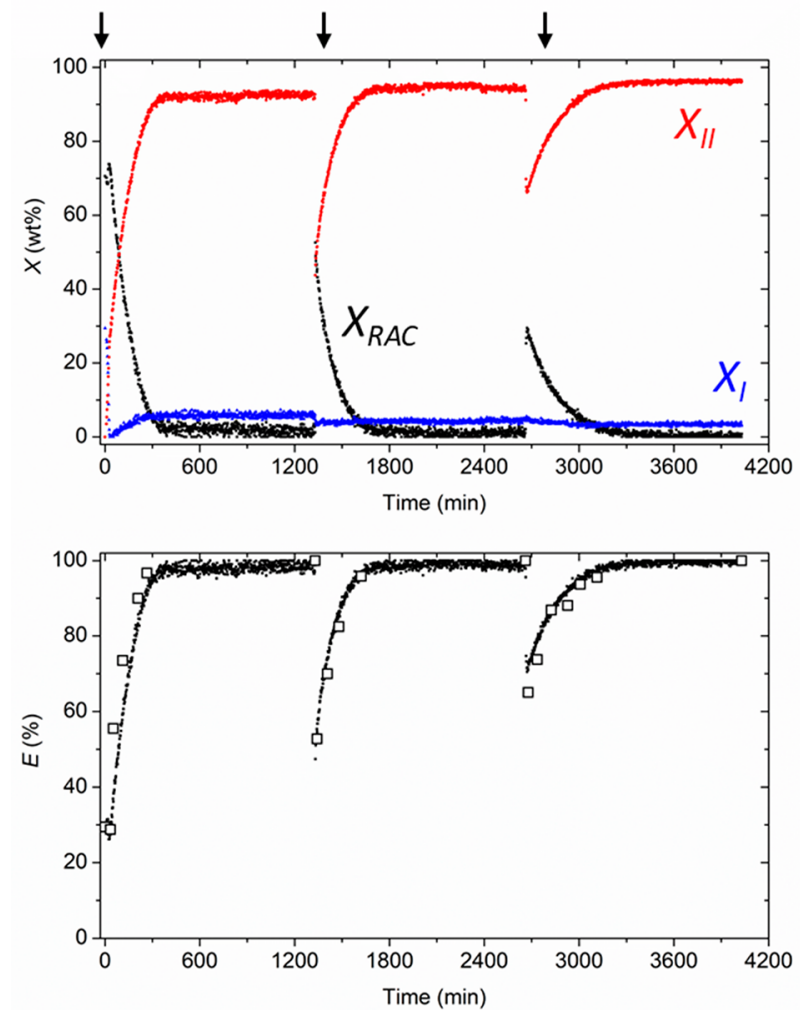

SB2
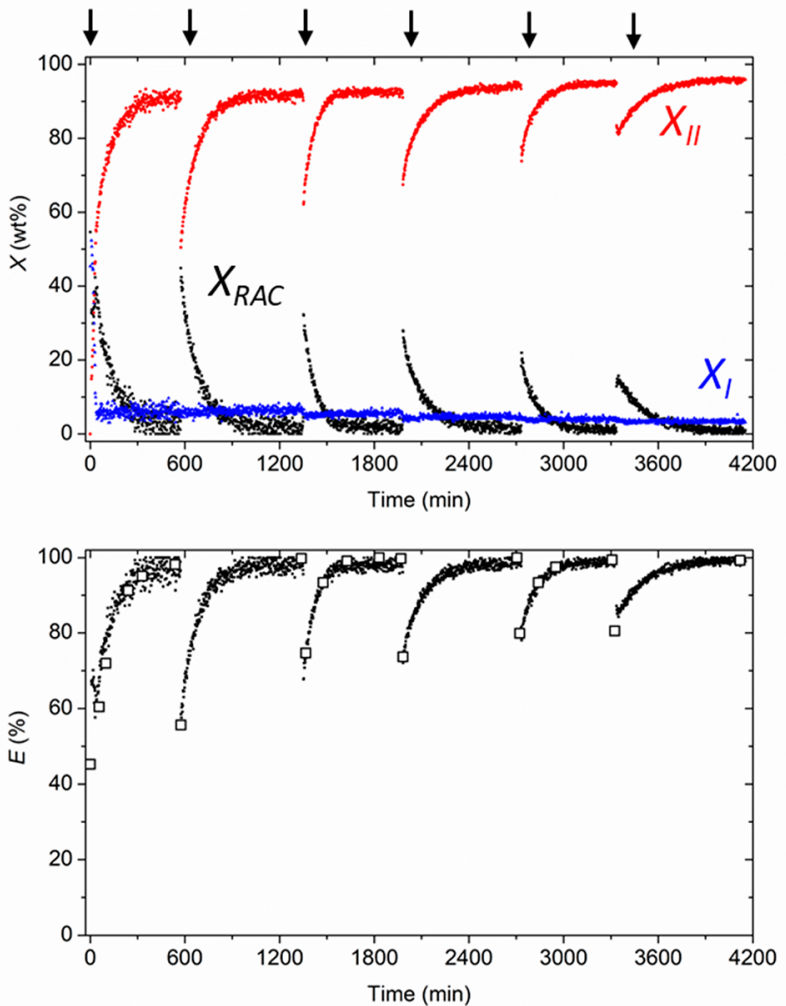

Figure 6. (top row) In situ Raman monitoring of the temporal evolution of the solid phase fractions of NCPA during semibatch deracemization experiments (SB1 and SB2, Table 2). (bottom row) Comparison of enantiomeric excess (open points) with Raman form I and II fractions (dots). Arrows indicate the points of racemic compound addition ( $1.92 \mathrm{~g} /$ addition for SB1 and $0.96 \mathrm{~g} / \mathrm{addition}$ for SB2).

fractions present at any time, as measured by Raman, against the actual solid-phase enantiomeric excess measured offline by chiral HPLC. While experiment B1 results in the racemic compound and unsuccessful deracemization, experiment B4 results in conglomerate crystals and complete deracemization at the end of the process. Overall, a very good agreement is obtained between the enantiomeric excess determined from the sum of the form I and II fractions and that from HPLC, demonstrating the ability of Raman spectroscopy to monitor in real time the deracemization process under the experimental conditions studied here.

However, it is seen that the Raman data consistently slightly overestimate the actual enantiomeric excess, which indicates that formation of the counter enantiomer may take place to some small extent. The combination of the form I and II fractions $X_{\mathrm{I}}$ and $X_{\mathrm{II}}$ from Raman and the enantiomeric excess $E$ determined from HPLC results for the offline samples allows the calculation of the total fraction of counter enantiomer $(S)$ present as form I and II crystals during the process according to the equation

$$
X_{S, \text { con }}=\frac{X_{\mathrm{I}}+X_{\mathrm{II}}-E}{2}
$$

Figure 5 (right) shows the evolution of the counter enantiomer form I and II fractions formed during experiments B1-B4. For experiments B3 and B4 that led to enantiopurity, the amount of counter enantiomer formed increases initially up to a maximum $(\sim 6 \%)$ around the point when the racemic crystals completely dissolve (Figure 3), after which it starts to decrease. During the racemic compound dissolution phase, the concentration of both enantiomers in the liquid phase remains high, creating supersaturation with respect to both enantiomers of the less soluble form II. Since only the $R$ enantiomer is seeded, it crystallizes more quickly than the $S$ enantiomer, which leads to the conversion of some supersaturation of the $S$ enantiomer to supersaturation for the $R$ enantiomer through the racemization. However, while dissolution of the racemic compound keeps occurring, the supersaturation for the $S$ enantiomer is continuously replenished, eventually leading to the formation of $S$ form II crystals. Once the racemic compound is depleted, the supersaturation for the $S$ enantiomer cannot be further sustained, which causes its formation to stop. Eventually, the small amount of $S$ enantiomer form II formed converts fully to $R$ enantiomer form II through Viedma ripening.

3.4. Semibatch Experiments. It is clear from the results presented in the previous sections that complete deracemization of NCPA is possible in batch experiments. In addition, Raman spectroscopy offers the ability to monitor in situ the temporal evolution of the solid-phase fractions in those experiments, which in this case gives an overall good online estimation of the enantiomeric excess throughout the process. However, it is seen that in many cases formation of counter enantiomer conglomerate crystals of form II can still occur. While these can still be converted to the preferred enantiomer through Viedma ripening, the process becomes slower. Thus, for an efficient deracemization process, it is preferable to suppress the formation of counter enantiomer crystals. In the batch experiments, this is possible by operating the process at relatively high solid loading and relatively high seed/racemic compound ratio in combination with grinding to ensure that 
crystallization of the seeded enantiomer takes place much faster than the crystallization of the counter enantiomer. However, operating under such conditions in batch leads to low productivity, as the amount of racemic compound to convert per batch is rather low, while increasing the solids loading requires the availability of a high amount of enantiopure seeds.

From a production standpoint, it thus seems preferable to start the process with a given (low) amount of enantiopure seeds and gradually feed small amounts of racemic compound, in a semibatch operation, allowing it to convert before a new addition. In this way, it is ensured that the supersaturation for the counter enantiomer remains low throughout the entire process, preventing the formation of counter enantiomer crystals. In addition, the system is auto seeded since the crystals of the preferred enantiomer accumulate in the reactor and provide ample surface area for the preferential crystallization of that enantiomer during subsequent additions, circumventing the need for large amounts of enantiopure seeds. Such a semibatch operation would be analogous to semibatch bioreactors, ${ }^{46}$ in which nutrients are intermittently added to prevent inhibitory actions present at high nutrient concentration or alteration of the product distribution (e.g., excessive ethanol production at high glucose concentrations during baker's yeast production ${ }^{46}$ ), and is a first step toward continuous deracemization. Moreover, semibatch deracemization could benefit substantially from Raman spectroscopy, as Raman may be used to monitor the evolution of the process and provide information on appropriate feeding strategies or even be used in conjunction with feedback control systems to fully automate the process.

In order to investigate the potential of semibatch deracemization, we carried out two additional experiments, SB1 and SB2 (see Table 2), in which the racemic compound was added gradually in the suspension. In both experiments, the same total amount of racemic compound ( $5.76 \mathrm{~g}$ in $20 \mathrm{~mL}$ of solvent) was added over a period of a little more than 3 days. In SB1, the racemic compound was added in three additions of $1.92 \mathrm{~g}$ each, approximately every $22 \mathrm{~h}$, while in SB2, it was added in six additions of $0.96 \mathrm{~g}$ approximately every $11 \mathrm{~h}$, leading to the same average addition rate of $\sim 1.4 \mathrm{mg} / \mathrm{min}$ of racemic compound. In both experiments, an amount of water (1.3 and $0.65 \mathrm{~g}$ for SB1 and SB2, respectively) was also added together with the racemic compound, leading to a gradual increase in the water concentration in the experiments. While water addition helps in enhancing the rate of crystal transformations, in some of our earlier experiments (not reported here) that were started at lower DBU concentration (1.2 wt \%), it was found that racemization stopped above a water concentration of $\sim 14 \mathrm{wt} \%$. This effect is most probably related to the deprotonation of water by DBU, which then becomes a weaker base and thus exhibits less catalytic activity. It was possible to circumvent this effect by increasing the concentration of DBU (3.4 wt \%), so as to maintain a $\mathrm{pH}$ of around 12 , as reported elsewhere. ${ }^{39}$

The evolution of the solid-phase fractions for experiments SB1 and SB2, measured by Raman spectroscopy, is reported in Figure 6 (top row). It is seen that Raman captures well the effect of racemic compound addition, with the racemate fraction increasing and the form II fraction decreasing upon every addition. It can also be seen that after a new addition of the racemic compound a fast and full transformation occurs to form II. A similar oscillating trend is observed in the solid-phase enantiomeric excess (Figure 6, bottom), where a sharp decrease is obtained right after the addition of the racemic compound, followed by an increase up to $E>99 \%$, as the racemic compound gradually converts to $R$ form II crystals after each addition. As expected, the decrease in the mass fraction of form II, and thus the enantiomeric excess, is less pronounced after each addition, as the form II crystals of the $R$ enantiomer build up in the reactor, leading to an increase in the suspension density.

Figure 6 also shows that the evolution of the enantiomeric excess during the semibatch process is directly estimated in real time by the Raman fractions with very good accuracy, possibly eliminating the need for manual sampling in those experiments. This in turn indicates that, as expected during the semibatch experiments, crystallization of the counter enantiomer is unlikely to occur, as the system remains well-seeded by the preferred enantiomer throughout the process. Table 2 shows the yields for the experiments, which are similar and on the order of $\sim 80 \%$ of the theoretical yield, with losses pertaining most probably due to sampling and filtration, indicating that the semibatch crystal transformation process is a feasible approach to obtain enantiopure solids.

\section{DISCUSSION}

While the usefulness of PATs in the design and control of crystallization processes has been demonstrated with considerable success, their use in chiral crystallization and deracemization has generally been difficult. By exploiting the ability of Raman spectroscopy to capture the different packings of the racemic compound and conglomerate crystals, we have demonstrated that online Raman spectroscopy can be used to quantify the solid-phase fractions during deracemization experiments in which various crystal structures (conglomerate and racemic compound) are involved.

On the basis of the temporal evolution of the solid-phase fractions enabled by Raman and the derived phase diagram for the studied NCPA system, it was possible to elucidate the crystal transformations possible under the various operating conditions and their influence on deracemization (Figure 7). In the absence

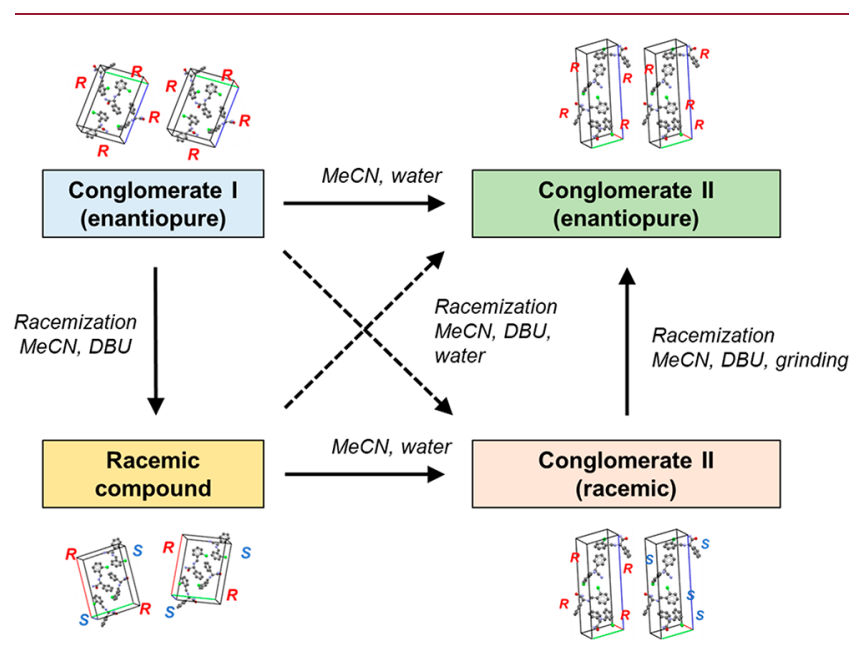

Figure 7. Possible transformation pathways between the various NCPA crystal structures in acetonitrile in the presence of water and/or racemization catalyst (DBU).

of racemization, starting from form I crystals of a single enantiomer, one can obtain form II crystals of the same enantiomer in an acetonitrile-water system. However, when racemization is enabled by DBU, form I converts first to the racemic compound in a pure acetonitrile-DBU system or directly to racemic conglomerate form II in an acetonitrile- 
water-DBU system. On the other hand, the racemic compound may directly convert to form II crystals of a single enantiomer or racemic conglomerate form II in the presence and absence of racemization, respectively. Finally, it is possible to convert racemic conglomerate form II crystals to form II crystals of a single enantiomer by grinding in the presence of racemization (Viedma ripening). By careful management of the experimental process parameters (racemization, amount of water) one can control the crystal transformations involved and achieve complete deracemization for this complex system.

Importantly, the results reveal that, for many of these deracemization experiments, knowledge of the evolution of the solid-phase composition can be used to infer the evolution of the solid-phase enantiomeric excess with good accuracy. However, it is noted that prediction of the enantiomeric excess using Raman data can only be achieved in highly specific cases, such as that reported in this work, which combine conglomerate crystallization, solution racemization, and such polymorphic behavior. Additionally, since Raman cannot distinguish between conglomerate crystals of different chiralities, the method fails if a substantial amount of the counter enantiomer conglomerate crystals is formed. While in those cases additional offline measurement is still needed to monitor the deracemization process, the Raman data remain useful, as their combination with the offline enantiomeric excess measurements can provide information on the kinetics of the undesired enantiomer formation. Overall, the results of this work emphasize the usefulness of in situ Raman spectroscopy in the development of complex (continuous) chiral crystallization processes involving various types of crystalline racemates. Although here we focused on deracemization through a metastable racemic compound to conglomerate transformation, we believe that in situ Raman spectroscopy may prove useful in other chiral resolution methods involving various types of crystalline racemates, such as preferential crystallization of racemic compound forming systems at the eutectic point. ${ }^{47}$

Finally, the promising results obtained from the semibatch experiments may indicate that a fully continuous process could be an achievable and possibly advantageous operating mode for deracemization. For the system studied in this work, such a process could feature continuous feeding of the racemic compound crystals and continuous product removal followed by in-line filtration and mother liquor recycle. Since in theory such a process can be operated at a steady state where the deracemization rate is maintained constant at high levels and it also circumvents nonproductive downtime that is required for collecting the solids, cleaning the reactor, and preparing a new batch, the productivity could possibly be higher than the batch process.

\section{CONCLUSION}

We achieved complete deracemization of $\mathrm{N}$-(2chlorobenzylidene)phenylglycine amide (NCPA), a complex chiral polymorphic system, by exploiting the solvent-mediated transformation of a racemic compound to a stable conglomerate. This process, also involving a metastable conglomerate, was monitored by Raman spectroscopy, which showed the usefulness of achiral PAT in this deracemization process. The phase diagram shows that under racemic conditions in the presence of the racemizing agent the racemic compound is just slightly more stable than the metastable conglomerate I. Slight enrichments in the solution in the absence of the racemizing agent promote the formation of enantiopure crystals of the enriched form. It turns out that a small water content has a substantial effect on how the deracemization process proceeds as well as the successfulness of the deracemization. The semibatch experiments with intermittent addition of the racemic compound to the transforming suspension show that this is a beneficial operation in comparison to batchwise operation. It is therefore envisaged that a continuous deracemization process for this compound may well be achievable.

\section{ASSOCIATED CONTENT}

\section{Supporting Information}

The Supporting Information is available free of charge on the ACS Publications website at DOI: 10.1021/acs.cgd.9b00867.

Synthesis procedure of the starting materials, NMR, HPLC, and XRPD data of the synthesized materials, calibration of Raman spectra, chemical stability experiment of NCPA in water, and a comparison between online Raman measurements in a suspension and offline data of the samples taken after filtration and drying of the crystals for all reported experiments (PDF)

\section{AUTHOR INFORMATION}

\section{Corresponding Authors}

*E-mail for C.X.: christos.xiouras@kuleuven.be.

*E-mail for H.M.: h.meekes@science.ru.nl.

*E-mail for E.V.: e.vlieg@science.ru.nl.

*E-mail for G.D.S.: georgios.stefanidis@kuleuven.be.

*E-mail for J.H.T.H.: joop.terhorst@strath.ac.uk.

\section{ORCID}

Christos Xiouras: 0000-0001-7162-234X

Alison Nordon: 0000-0001-6553-8993

Hugo Meekes: 0000-0001-9236-2129

Elias Vlieg: 0000-0002-1343-4102

Georgios D. Stefanidis: 0000-0002-4347-1350

Joop H. Ter Horst: 0000-0003-0118-2160

Notes

The authors declare no competing financial interest.

\section{ACKNOWLEDGMENTS}

C.X. acknowledges funding of a Ph.D. fellowship by the Research Foundation-Flanders. (FWO). C.X. and G.B. acknowledge the hospitality they received during their research visit to CMAC at the University of Strathclyde to perform part of this research. This research received funding as part of the CORE project (October 2016-September 2020) from the European Union's Horizon 2020 research and innovation programme under the Marie Sklodowska-Curie grant agreement No. 722456 CORE ITN. J.H.T.H. and R.V. thank the EPSRC Centre for Innovative Manufacturing in Continuous Manufacturing and Crystallization (http://www.cmac.ac.uk) for supporting this work (EPSRC funding under Grant Reference: EP/I033459/1).

\section{REFERENCES}

(1) Viedma, C. Chiral symmetry breaking during crystallization: Complete chiral purity induced by nonlinear autocatalysis and recycling. Phys. Rev. Lett. 2005, 94, 3-6.

(2) Suwannasang, K.; Flood, A. E.; Rougeot, C.; Coquerel, G. Using programmed heating-cooling cycles with racemization in solution for complete symmetry breaking of a conglomerate forming system. Cryst. Growth Des. 2013, 13, 3498-3504.

(3) McBride, J. M.; Tully, J. C. Did life grind to a start? Nature 2008, $452,161-162$. 
(4) Blackmond, D. G. "Chiral amnesia" as a driving force for solidphase homochirality. Chem. - Eur. J. 2007, 13, 3290-3296.

(5) Uwaha, M.; Katsuno, H. Mechanism of chirality conversion by grinding crystals: Ostwald ripening vs crystallization of chiral clusters. $J$. Phys. Soc. Jpn. 2009, 78, 023601.

(6) Noorduin, W. L.; Van Enckevort, W. J. P.; Meekes, H.; Kaptein, B.; Kellogg, R. M.; Tully, J. C.; McBride, J. M.; Vlieg, E. The driving mechanism behind attrition-enhanced deracemization. Angew. Chem., Int. Ed. 2010, 49, 8435-8438.

(7) Viedma, C.; Ortiz, J. E.; De Torres, T.; Izumi, T.; Blackmond, D. G. Evolution of Solid Phase Homochirality for a Proteinogenic Amino Acid Evolution of Solid Phase Homochirality for a Proteinogenic Amino Acid. J. Am. Chem. Soc. 2008, 130, 15274-15275.

(8) Spix, L.; Meekes, H.; Blaauw, R. H.; Van Enckevort, W. J. P.; Vlieg, E. Complete deracemization of proteinogenic glutamic acid using viedma ripening on a metastable conglomerate. Cryst. Growth Des. 2012, 12, 5796-5799.

(9) Wilmink, P.; Rougeot, C.; Wurst, K.; Sanselme, M.; Van Der Meijden, M.; Saletra, W.; Coquerel, G.; Kellogg, R. M. Attrition induced deracemisation of 2-fluorophenylglycine. Org. Process Res. Dev. 2015, 19, 302-308.

(10) Kaptein, B.; Noorduin, W. L.; Meekes, H.; Van Enckevort, W. J. P.; Kellogg, R. M.; Vlieg, E. Attrition-enhanced deracemization of an amino acid derivative that forms an epitaxial racemic conglomerate. Angew. Chem., Int. Ed. 2008, 47, 7226-7229.

(11) Kawasaki, T.; Takamatsu, N.; Aiba, S.; Tokunaga, Y. Spontaneous formation and amplification of an enantioenriched $\alpha$ amino nitrile: A chiral precursor for Strecker amino acid synthesis. Chem. Commun. 2015, 51, 14377-14380.

(12) Kellogg, R.; Van der Meijden, M.; Leeman, M.; Gelens, E.; Noorduin, W.; Meekes, H.; Van Enckevort, W.; Kaptein, B.; Vlieg, E. Attrition-Enhanced Deracemization in the Synthesis of Clopidogrel-A Practical Application of a New Discovery. Org. Process Res. Dev. 2009, 13, 1195-1198.

(13) Noorduin, W. L.; Kaptein, B.; Meekes, H.; Van Enckevort, W. J. P.; Kellogg, R. M.; Vlieg, E. Fast attrition-enhanced deracemization of naproxen by a gradual in situ feed. Angew. Chem., Int. Ed. 2009, 48, $4581-4583$.

(14) Björemark, P. M.; Jönsson, J.; Håkansson, M. Absolute Asymmetric Synthesis: Viedma Ripening of [Co(bpy) 3 ] 2+ and Solvent-Free Oxidation to [Co(bpy) 3 ] 3+. Chem. - Eur. J. 2015, 21, 10630-10633.

(15) Engwerda, T.; Mertens, H.; Tinnemans, P.; Meekes, H.; Rutjes, F. P. J. T.; Vlieg, E. Solid-Phase Conversion of Four Stereoisomers into a Single Enantiomer. Angew. Chem., Int. Ed. 2018, 57, 15441-15444.

(16) Bernstein, J. Polymorphism in Molecular Crystals; Oxford University Press: 2002.

(17) Jacques, J.; Collet, A.; Wilen, S. Enantiomers, Racemates and Resolutions; Krieger: 1981.

(18) Xiouras, C.; Van Cleemput, E.; Kumpen, A.; Ter Horst, J. H.; Van Gerven, T.; Stefanidis, G. D. Towards deracemization in the absence of grinding through crystal transformation, ripening, \& racemization. Cryst. Growth Des. 2017, 17, 882-890.

(19) Engwerda, A. H. J.; Meekes, H.; Kaptein, B.; Rutjes, F. P. J. T.; Vlieg, E. Speeding up Viedma ripening. Chem. Commun. 2016, 52, 12048-12051.

(20) Xiouras, C.; Ter Horst, J. H.; Van Gerven, T.; Stefanidis, G. D. Coupling Viedma Ripening with Racemic Crystal Transformations: Mechanism of Deracemization. Cryst. Growth Des. 2017, 17, 49654976.

(21) Cardew, P. T.; Davey, R. J. Proc. R. Soc. London, Ser. A 1985, 398, 415-428.

(22) Nagy, Z. K.; Fevotte, G.; Kramer, H.; Simon, L. L. Recent advances in the monitoring, modelling and control of crystallization systems. Chem. Eng. Res. Des. 2013, 91, 1903-1922.

(23) Févotte, G. In situ Raman spectroscopy for in-line control of pharmaceutical crystallization and solids elaboration processes: A review. Chem. Eng. Res. Des. 2007, 85, 906-920.
(24) Wong, S.; Georgakis, C.; Botsaris, G. D.; Saranteas, K.; Bakale, R. Online Estimation of Diastereomer Composition Using Raman: Differentiation in High and Low Slurry Density Partial Least Square Models. Cryst. Growth Des. 2008, 8, 4398-4408.

(25) Mathieu, J. P. Vibration Spectra and Polymorphism of Chiral Compounds. J. Raman Spectrosc. 1973, 1, 47-51.

(26) Inoue, M.; Hisada, H.; Koide, T.; Carriere, J.; Heyler, R.; Fukami, T. In Situ Monitoring of Crystalline Transformation of Carbamazepine Using Probe-Type Low-Frequency Raman Spectroscopy. Org. Process Res. Dev. 2017, 21, 262-265.

(27) Wang, X.; Watcher, J. A.; Antosz, F. J.; Berglund, K. A. Org. Process Res. Dev. 2000, 4, 391-395.

(28) Caillet, A.; Fevotte, G. Quantitative in situ monitoring of citric acid phase transition in water using Raman spectroscopy. Chem. Eng. Process. 2008, 47, 377-382.

(29) Starbuck, C.; Spartalis, A.; Wai, L.; Wang, J.; Fernandez, P.; Lindemann, C. M.; Zhou, G. X.; Ge, Z. Process Optimization of a Complex Pharmaceutical Polymorphic System via In Situ Raman Spectroscopy. Cryst. Growth Des. 2002, 2, 515-522.

(30) Jiang, S.; Jansens, P. J.; Ter Horst, J. H. Mechanism and kinetics of the polymorphic transformation of o-aminobenzoic acid. Cryst. Growth Des. 2010, 10, 2123-2128.

(31) Schöll, J.; Bonalumi, D.; Vicum, L.; Mazzotti, M.; Mueller, M. Cryst. In Situ Monitoring and Modeling of the Solvent-Mediated Polymorphic Transformation of L-Glutamic Acid. Cryst. Growth Des. 2006, 6, 881-891.

(32) Esmonde-White, K. A.; Cuellar, M.; Uerpmann, C.; Lenain, B.; Lewis, I. R. Raman spectroscopy as a process analytical technology for pharmaceutical manufacturing and bioprocessing. Anal. Bioanal. Chem. 2017, 409, 637-649.

(33) Simone, E.; Saleemi, A. N.; Nagy, Z. K. Application of quantitative Raman spectroscopy for the monitoring of polymorphic transformation in crystallization processes using a good calibration practice procedure. Chem. Eng. Res. Des. 2014, 92, 594-611.

(34) Cornel, J.; Lindenberg, C.; Mazzotti, M. Quantitative application of in-situ ATR-FTIR and Raman spectroscopy in crystallization processes. Ind. Eng. Chem. Res. 2008, 47, 4870-4882.

(35) Braun, D. E.; Maas, S. G.; Zencirci, N.; Langes, C.; Urbanetz, N. A.; Griesser, U. J. Simultaneous quantitative analysis of ternary mixtures of d -mannitol polymorphs by FT-Raman spectroscopy and multivariate calibration models. Int. J. Pharm. 2010, 385, 29-36.

(36) Farias, M.; Carneiro, R. Simultaneous Quantification of Three Polymorphic Forms of Carbamazepine in the Presence of Excipients Using Raman Spectroscopy. Molecules 2014, 19, 14128-14138.

(37) Dandeu, A.; Humbert, B.; Carteret, C.; Muhr, H.; Plasari, E.; Bossoutrot, J. M. Raman spectroscopy - A powerful tool for the quantitative determination of the composition of polymorph mixtures: Application to $\mathrm{CaCO} 3$ polymorph mixtures. Chem. Eng. Technol. 2006, $29,221-225$.

(38) George, F.; Norberg, B.; Wouters, J.; Leyssens, T. Structural Investigation of Substituent Effect on Hydrogen Bonding in (S)Phenylglycine Amide Benzaldimines. Cryst. Growth Des. 2015, 15, 4005-4019.

(39) Noorduin, W. L.; Izumi, T.; Millemaggi, A.; Leeman, M.; Meekes, H.; Van Enckevort, W. J. P.; Kellogg, R. M.; Kaptein, B.; Vlieg, E.; Blackmond, D. G. Emergence of a single solid chiral state from a nearly racemic amino acid derivative. J. Am. Chem. Soc. 2008, 130, $1158-1159$.

(40) Rinnan, A.; Van den Berg, F.; Balling Engelsen, S. Review of the most common pre-processing techniques for near-infrared spectra. TrAC, Trends Anal. Chem. 2009, 28, 1201-1222.

(41) De Jong, S. SIMPLS: An alternative approach to partial least squares regression. Chemom. Intell. Lab. Syst. 1993, 18, 251-263.

(42) Khoshkhoo, S.; Anwar, J. J. Crystallization of polymorphs: the effect of solvent. J. Phys. D: Appl. Phys. 1993, 26, B90-B93.

(43) Nicoud, L.; Licordari, F.; Myerson, A. S. Estimation of the solubility of metastable polymorphs. A critical review. Cryst. Growth Des. 2018, 18, 7228-7237. 
(44) Granberg, R. A.; Rasmuson, C. Solubility of Paracetamol in Binary and Ternary Mixtures of Water + Acetone + Toluene. J. Chem. Eng. Data 2000, 45, 478-483.

(45) Gu, C. H.; Young, V.; Grant, D. J. W. Polymorph screening: Influence of solvents on the rate of solvent-mediated polymorphic transformation. J. Pharm. Sci. 2001, 90, 1878-1890.

(46) Yamanè, T.; Shimizu, S. Fed-batch techniques in microbial processes. In: Bioprocess Parameter Control. Advances in Biochemical Engineering/Biotechnology, 1984, vol 30. Springer, Berlin, Heidelberg. (47) Gou, L.; Robl, S.; Leonhard, K.; Lorenz, H.; Sordo, M.; Butka, A.; Kesselheim, S.; Wolff, M.; Seidel-Morgenstern, A.; Schaber, K. A hybrid process for chiral separation of compound-forming systems. Chirality 2011, 23, 118-127. 